\title{
Temporal feeding habits of the whitemouth croaker Micropogonias furnieri in a shallow coastal lagoon (southwestern Atlantic Ocean, Uruguay)
}

\section{Hábitos temporales de alimentación de la corvina blanca Micropogonias furnieri en una laguna costera poco profunda (océano Atlántico sudoccidental, Uruguay)}

\author{
Daniela Olsson ${ }^{1}$, Florencia Forni ${ }^{1}$, Gustavo Saona ${ }^{1}$, José Verocai ${ }^{2}$, Walter Norbis ${ }^{1 *}$ \\ ${ }^{1}$ Laboratorio de Fisiología de la Reproducción y Ecología de Peces, Departamento de Biologia Animal, \\ Instituto de Biología, Facultad de Ciencias, Universidad de la República, Iguá 4225, Montevideo, Uruguay. \\ 2 Sección de Oceanología, Instituto de Ecología y Ciencias Ambientales, Facultad de Ciencias, Universidad de \\ la República, Iguá 4225, Montevideo, Uruguay.
}

* Corresponding author. E-mail: walter.norbis@gmail.com

\begin{abstract}
We analyzed the stomach contents of 2915 individuals of the whitemouth croaker, Micropogonias furnieri, ranging from 15.6 to $42.0 \mathrm{~cm}$ total length, collected from a coastal lagoon in Rocha, Uruguay, during 1997-1999. A total of 56\% ( $n=1646)$ of individuals were found with content in their stomachs. Six food items were identified and classified as crustaceans (49\%), polychaetes (26\%), vegetal debris (15\%), molluscs, juvenile fishes, and unidentified contents, the latter three representing less than $5 \%$ of the total stomach contents. Fishes displayed a higher proportion of stomachs with food between February and August 1998, simultaneous with a low gonadosomatic index and a decrease in water temperature. Crustaceans were the dominant food item from November 1997 to March 1998 and from November to December 1998 (austral summer), whereas polychaetes were predominant from April to October 1998 and from January to February 1999. Availability of polychaetes and their higher frequency of occurrence in the stomachs before the reproductive period could play an important role in the reproduction of the species. Feeding frequency was correlated with length classes. Smaller fishes $(<25 \mathrm{~cm})$ preyed on polychaetes and vegetal debris, whereas larger fishes fed mainly on crustaceans and fishes. The temporal feeding habits of M. furnieri in this coastal lagoon showed selective and opportune utilization of prey items according to annual variability in reproductive status and water temperature.
\end{abstract}

Key words: feeding, whitemouth croaker, coastal lagoon, Uruguay.

RESUMEN. Fueron analizados los contenidos estomacales de 2915 individuos de la corvina blanca, Micropogonias furnieri, entre 15.6 y $42.0 \mathrm{~cm}$ longitud total, recolectados en la laguna costera de Rocha, Uruguay, de 1997 a 1999 . El 56\% de los individuos ( $n=1646)$ fue encontrado con contenido en sus estómagos. Se identificaron seis ítems alimenticios y se clasificaron como crustáceos (49\%), poliquetos (26\%), restos vegetales (15\%), moluscos, juveniles de peces, y restos no identificados (éstos últimos representaron menos que el 5\% del total de contenidos estomacales). Se encontró una gran proporción de estómagos con alimento de febrero a agosto de 1998, en simultaneo con bajos índices gonadosomáticos y un descenso en la temperatura del agua. Los crustáceos predominaron como ítem alimenticio desde noviembre de 1997 hasta marzo de 1998 y de noviembre a diciembre de 1998 (verano austral), mientras que los poliquetos predominaron desde abril hasta octubre de 1998 y en enero y febrero de 1999. La disponibilidad de poliquetos y su mayor frecuencia de ocurrencia en los estómagos antes del periodo reproductivo podría jugar un rol importante en la reproducción de la especie. La frecuencia de alimentación estuvo correlacionada con las clases de talla. Los peces más pequeños $(<25 \mathrm{~cm})$ depredaron sobre poliquetos y restos vegetales, mientras que los peces más grandes se alimentaron principalmente de crustáceos y peces. Los hábitos de alimentación temporales de $M$. furnieri en esta laguna costera mostraron una utilización selectiva y oportuna de los ítems presa a lo largo del año, de acuerdo con el ciclo de reproducción de la especie y la variación de la temperatura.

Palabras clave: alimentación, corvina blanca, laguna costera, Uruguay.

\section{INTRODUCTION}

Ecosystem modelling approach, which takes multispecies relationships into account, requires the knowledge of trophic interactions among constituent organisms (Palomares and Pauly 1999, Bozec et al. 2004). Such information can be difficult to obtain for individual species that constitute the object of analysis in the system. The food and feeding relationships among fishes in the coastal lagoons of the

\section{INTRODUCCIÓN}

Los estudios orientados al modelado de ecosistemas, que toman en cuenta muchas especies, requieren del conocimiento de los requerimientos alimenticios e interacciones tróficas de los organismos constituyentes (Palomares y Pauly 1999, Bozec et al. 2004). Tal conocimiento es a menudo difícil de obtener para una especie que constituye el objeto de análisis en el sistema. Las relaciones entre el alimento y la 
southwestern Atlantic Ocean, particularly of Uruguay, are poorly documented (Araujo 1984, Castello 1985, Norbis and Galli 2004, Rodríguez-Graña et al. 2008).

The whitemouth croaker, Micropogonias furnieri (Desmarest 1823), is a demersal euryhaline coastal species. Its distribution extends from the Yucatán Peninsula (Mexico, $28^{\circ} \mathrm{N}$ ), along the southern Caribbean and the eastern South American coast, to the Gulf of San Matías (Argentina, $41^{\circ} \mathrm{S}$ ) (Isaac 1988). The species is particularly abundant in the Río de la Plata Basin and serves as the principal resource in the coastal and artisanal fisheries of Uruguay and Argentina (Arena 1990, Norbis 1995). The broad distribution of the whitemouth croaker is a result of its high adaptability, in particular with regard to its feeding habits. Many studies have analyzed the food and feeding habits of this species in estuaries and the continental shelf (Puig 1986, Sánchez et al. 1991, Masello et al. 2002, Chaves and Umbria 2003, Mandali and Paes 2005, Mendoza-Carranza and Vieira 2008), marine surf zone (Texeira et al. 1992), and coastal lagoons (Mandali and Paes 1998, Hozbor and García de la Rosa 2000), and some have considered population characteristics (Goncalvez et al. 1999, Mandali and Paes 1998), but none have analyzed temporal variations in feeding habits. The whitemouth croaker has a seasonal reproductive cycle synchronized with annual oscillations in temperature, whereby the spawning period occurs during the warm phase and the resting period during the cold phase (Vizziano 2002, Vizziano et al. 2002). Food availability during the autumn and winter (Southern Hemisphere) may be critical for optimal maturation and reproduction of whitemouth croaker in coastal lagoons. The aim of this study was to analyze the temporal variation (monthly) in feeding habits of $M$. furnieri in a shallow coastal lagoon of the southwestern Atlantic Ocean, and explore its relationship with both its reproductive cycle and environmental variability.

\section{MATERIALS AND METHODS}

Rocha Lagoon is a shallow (mean depth $=0.6 \mathrm{~m}$; area $=$ $72 \mathrm{~km}^{2}$ ), brackish, microtidal lagoon within a drainage area of approximately $1216 \mathrm{~km}^{2}$, located on the southwestern coast of South America ( $34^{\circ} 33^{\prime}$ S, $54^{\circ} 22^{\prime}$ W) and part of the $\mathrm{MaB} / \mathrm{UNESCO}$ Biosphere Reserve (fig. 1). The lagoon is separated from the ocean by a sand bar parallel to the coast, which occasionally opens due to the action of southern storms (wind-driven effect), pulses of freshwater inputs (as a consequence of rain) or human actions. The wind-driven effect causes a strong salinity gradient within the lagoon (Pintos et al. 1991, Conde et al. 2000).

Sampling of the whitemouth croaker was carried out between November 1997 and February 1999 (table 1) in the southern area of the lagoon near the sand bar where hydrodynamic characteristics are more influenced by marine coastal waters (fig. 1) and where the species has a higher frequency of occurrence and abundance. Water salinity and temperature alimentación en peces en las lagunas costeras del océano Atlántico sudoccidental, particularmente en Uruguay, están poco documentadas (Araujo 1984, Castello 1985, Norbis y Galli 2004, Rodríguez-Graña et al. 2008).

La corvina blanca, Micropogonias furnieri (Desmarest 1823), es una especie demersal eurihalina costera. Su distribución se extiende desde la península de Yucatán (México, $28^{\circ} \mathrm{N}$ ), a lo largo de la costa sur del Caribe y de la costa este de Sudamérica, hasta el golfo de San Matías (Argentina, $41^{\circ} \mathrm{S}$ ) (Isaac 1988). La especie es particularmente abundante en el Río de la Plata y es el principal recurso para las pesquerías costeras y artesanales de Uruguay y Argentina (Arena 1990, Norbis 1995). La amplia distribución de la corvina blanca es el resultado de su gran adaptabilidad, en particular en sus hábitos de alimentación. Muchos autores han analizado el alimento y los hábitos alimenticios de esta especie en estuarios y en la plataforma continental (Puig 1986, Sánchez et al. 1991, Masello et al. 2002, Chaves y Umbria 2003, Mandali y Paes 2005, Mendoza-Carranza y Vieira 2008), la zona de rompiente marina (Texeira et al. 1992) y las lagunas costeras (Mandali y Paes 1998, Hozbor y Garcia de la Rosa 2000), y algunos han considerando las características de la población (Goncalvez et al. 1999, Mandali y Paes 1998), pero ninguno ha analizado los cambios en los hábitos alimenticios en el tiempo. La corvina blanca tiene un ciclo de reproducción sincronizado con oscilaciones anuales en la temperatura, con un período de desove durante la fase cálida y un período de reposo durante la fase fría (Vizziano 2002, Vizziano et al. 2002). La disponibilidad de alimento durante el otoño y el invierno (hemisferio Sur) puede ser crítica para una maduración y una reproducción óptima de la corvina blanca dentro de la laguna. El objetivo de este trabajo fue analizar los hábitos alimenticios temporales (por mes) de la corvina blanca en una laguna costera poco profunda del océano Atlántico sudoccidental, y explorar sus relaciones con el ciclo reproductivo y la variabilidad ambiental.

\section{MATERIALES Y MÉTODOS}

La laguna de Rocha es poco profunda (profundidad media $=0.6 \mathrm{~m}$; área $=72 \mathrm{~km}^{2}$ ), salobre y micromareal, se encuentra dentro de una cuenca de aproximadamente $1216 \mathrm{~km}^{2}$, ubicada en la costa suroeste de Sudamérica $\left(34^{\circ} 33^{\prime} \mathrm{S}, 54^{\circ} 22^{\prime} \mathrm{W}\right)$, y es parte de la reserva de la biósfera MaB/UNESCO (fig. 1). La laguna está separada del océano por una barra de arena paralela a la costa, la cual se abre ocasionalmente debido a la acción de las tormentas del sur (gobernado por efecto del viento), aportes de agua dulce (como consecuencia de la lluvia) o acciones humanas. El efecto del viento causa un fuerte gradiente de salinidad dentro de la laguna (Pintos et al. 1991, Conde et al. 2000).

Los muestreos de la corvina fueron realizados entre noviembre de 1997 y febrero de 1999 (tabla 1) en el área sur de la laguna cerca de la barra de arena donde las características hidrodinámicas están mas influenciadas por las aguas 


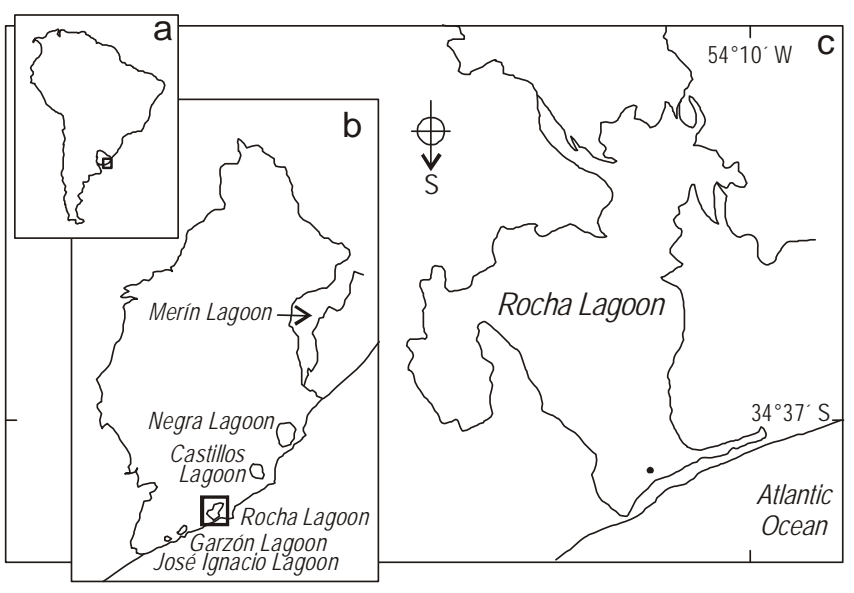

Figure 1. (a) Location of Uruguay in South America. (b) Bañados del Este Biosphera Reserve, with the system of coastal lagoons. (c) Rocha Lagoon (indicating the sampling point) (taken from Saona et al. 2003).

Figura 1. (a) Ubicación de Uruguay en Sudamérica. (b) Reserva de la Biósfera Bañados del Este, con el sistema de lagunas costeras. (c) Laguna de Rocha (se indica el punto de muestreo) (tomado de Saona et al. 2003).

were recorded monthly at the same point where individuals were caught. These parameters were measured with a multiparameter instrument (Yellow Springs Instruments, USA). Fish were caught at sunset using a beach seine (40 mm mesh in the wings and $10 \mathrm{~mm}$ at the cod ends, vertical opening of $1 \mathrm{~m}$, and $200 \mathrm{~m}$ length) trawled from $70-80 \mathrm{~cm}$ depth to the shoreline. This gear is considered the most appropriate for a shallow lagoon and it catches most of the length ranges of M. furnieri in Rocha Lagoon (Saona et al. 2003). Total length (TL) of all individuals caught was measured to the nearest millimeter for both sexes. Intact stomachs with contents were removed, transferred to the laboratory, and examined in relatively fresh condition.

Food items were identified to the lowest possible taxon using a dissecting microscope, with the exception of Polychaeta. Polychaetes were identified to species level but considered as a single group for the analysis. Prey items were grouped into higher taxonomic categories (e.g., crustaceans, molluscs, polychaetes, fishes, vegetal debris, and unidentified items) for comparative purposes (table 2). The number of empty stomachs was also recorded to compare the proportion of fish with and without stomach contents within each month of capture. A chi-squared test (Sokal and Rohlf 1995) was used to determine non-significant differences $(\alpha=0.05)$ between the proportion of stomachs with and without food for each month.

Feeding intensity was calculated per month using a feeding index (FI) (Hureau 1970), defined as: FI $=f_{s} /\left(f_{s}+e_{s}\right)$, where $f_{s}$ is the number of stomachs with food content and $e_{s}$ is the number of empty stomachs sampled. For each prey item the frequency of occurrence (\%Fi) was calculated: $\mathrm{Fi}=100 \times$ $\left(s_{i} / S_{c}\right)$ (Hyslop 1980), where $s_{i}$ is the number of stomachs costeras marinas (fig. 1) y donde la especie presenta una mayor frecuencia de ocurrencia y abundancia. La salinidad y la temperatura del agua fueron registradas mensualmente en el mismo punto donde se realizó la captura de los peces. Estos parámetros fueron medidos con un multiparámetro (Yellow Springs Instruments, Estados Unidos). Los peces fueron capturados al atardecer utilizando una red de arrastre de playa (40 mm de malla en las alas y $10 \mathrm{~mm}$ en el copo, abertura vertical de $1 \mathrm{~m}$ y $200 \mathrm{~m}$ de longitud) operada desde una profundidad de 70-80 cm hasta la línea de costa. Esta red es la más apropiada para una laguna costera y captura la mayoría de los intervalos de longitud de la corvina blanca en la laguna Rocha (Saona et al. 2003). La longitud total (LT) de todos los individuos capturados fue medida al milímetro más cercano para ambos sexos. Los estómagos intactos con contenido fueron extraídos, trasladados al laboratorio y examinados en condiciones relativamente frescas.

Los ítems alimenticios se identificaron al mínimo taxón posible utilizando una lupa binocular, excepto para los poliquetos. Los poliquetos fueron identificados solamente para conocer la especie, pero se consideraron como un único grupo para los análisis. Los ítems presa fueron agrupados en categorías taxonómicas altas (crustáceos, moluscos, poliquetos, peces, restos vegetales e ítems no identificados) con propósitos comparativos (tabla 2). El número de estómagos vacíos fue también registrado para comparar la proporción de peces con o sin contenido estomacal en cada mes de captura. Se utilizó una prueba de chi-cuadrado (Sokal y Rohlf 1995) para determinar si no existían diferencias significativas $(\alpha=$ 0.05 ) entre la proporción de estómagos con y sin alimento por mes.

La intensidad de alimentación por mes fue calculada utilizando el índice de alimentación (FI) (Hureau, 1970), definido como: $\mathrm{FI}=f_{s} /\left(f_{s}+e_{s}\right)$, donde $f_{s}$ es el número de estómagos con contenido alimenticio y $e_{s}$ es el número de estómagos vacíos por muestreo. Para cada ítem presa, se calculó la frecuencia de ocurrencia (\%Fi): $\mathrm{Fi}=100 \times\left(s_{i} / S_{c}\right)$ (Hyslop, 1980), donde $s_{i}$ es el número de estómagos que contienen la presa $i$, (excepto para poliquetos) y $S_{c}$ es el número de estómagos con contenido. Utilizando el coeficiente de correlación no paramétrico $\left(r_{s}\right)$ de Spearmann (Sokal y Rohlf 1995), se analizaron las relaciones entre FI y el índice gonadosomático $\left(I_{\mathrm{G}}\right)$ (calculado como $I_{\mathrm{G}}=$ (peso gónada/peso corporal total) $\times$ 100) (Vizziano et al. 2002) y entre la temperatura y la salinidad en el punto de muestreo por mes.

Se aplicó la prueba de independencia G (Sokal y Rohlf 1995) a las frecuencias de ocurrencias absolutas de las categorías taxonómicas de las presas (crustáceos, moluscos, poliquetos, peces, restos vegetales e ítems no identificados) para conocer si el consumo de esos ítems fue dependiente de la talla de la corvina blanca. Para este propósito, las corvinas blancas fueron separadas en seis clases de talla $(1: 15-20 \mathrm{~cm}$; 2: $21-25 \mathrm{~cm}$; 3: $26-30 \mathrm{~cm} \mathrm{4:} \mathrm{31-35} \mathrm{cm;} \mathrm{5:} \mathrm{36-40} \mathrm{cm;}$ 6: $41-45 \mathrm{~cm}$ ) de acuerdo con Masello et al. (2002) con fines comparativos. Fue aplicado el índice de Morisita (Smith y 
Table 1. Sampling months and years, number of fishes sampled ( $n$ ), number of stomachs with food (SF), number of empty stomachs (SE), value of the chi-squared test $\left(\chi^{2}\right)$, and feeding index (FI) $(*=$ significant differences; see materials and methods).

Tabla 1. Meses y años de muestreo, número de peces muestreados (n), número de estómagos con alimento (SF), número de estómagos vacíos (SE), valor de la prueba de chi-cuadrado $\left(\chi^{2}\right)$, e índice de alimentación (FI) $*^{*}=$ diferencias significativas; ver materiales y métodos).

\begin{tabular}{|c|c|c|c|c|c|}
\hline Month/year & $n$ & $\mathrm{SF}$ & SE & $\chi^{2}$ & FI \\
\hline December 1997 & 289 & 149 & 140 & 0.28 & 0.52 \\
\hline February 1998 & 418 & 291 & 127 & $64.34 *$ & 0.70 \\
\hline March 1998 & 309 & 193 & 116 & $19.19 *$ & 0.62 \\
\hline May 1998 & 111 & 74 & 37 & $12.33^{*}$ & 0.67 \\
\hline June 1998 & 178 & 118 & 60 & $18.90 *$ & 0.66 \\
\hline July 1998 & 59 & 40 & 19 & $7.47^{*}$ & 0.68 \\
\hline August 1998 & 67 & 43 & 24 & $5.39 *$ & 0.64 \\
\hline September 1998 & 135 & 52 & 83 & $7.12 *$ & 0.39 \\
\hline January 1999 & 173 & 79 & 94 & 1.30 & 0.46 \\
\hline February 1999 & 59 & 37 & 22 & 3.81 & 0.63 \\
\hline Total & 2915 & 1646 & 1269 & & \\
\hline$\%$ & & 56.5 & 43.5 & & \\
\hline
\end{tabular}

containing the prey $i$ (except for polychaetes) and $S_{c}$ is the number of stomachs with food content. The relationship between FI and the gonadosomatic index $\left(I_{\mathrm{G}}\right)$ (calculated as $I_{\mathrm{G}}=($ gonad weight/total body weight $\left.) \times 100\right)($ Vizziano et al. 2002), as well as that between temperature and salinity at the sampling point per month were analyzed using Spearman non-parametric correlation coefficients $\left(r_{s}\right)$ (Sokal and Rohlf 1995).

To determine whether prey item consumption was dependent on total length of the whitemouth croaker, the independence G-test (Sokal and Rohlf 1995) was used to compare the absolute frequencies of occurrence of prey taxonomic categories (crustaceans, molluscs, polychaetes, fishes, vegetal debris, and unidentified items). For this purpose, whitemouth croakers were separated into six length classes (1: 15-20 cm; 2: 21-25 cm; 3: 26-30 cm; 4: 31-35 cm; 5: 36-40 cm; and 6: $41-45 \mathrm{~cm}$ ) according to Masello et al. (2002) for comparative purposes. The Morisita index (Smith and Zaret 1982) was applied to analyze diet overlap between sizes. This index ranges from zero to one, whereby values $>0.6$ are considered a biologically significant overlap (Langton 1982).

Matrices for frequency of occurrence of prey items by month (M1) and for length classes for each month (M2) were analyzed using a correspondence analysis (CA) (ter Braak and Smilauer 1998, Marshall and Elliott 1997). Frequency of occurrence data were log-transformed $(\ln (x+1))$ (Jackson,
Zaret 1982) para analizar la superposición de dieta entre las tallas. El intervalo de este índice va de cero a uno, y cuando el valor excede 0.6 puede ser considerado como una superposición biológica significativa (Langton 1982).

Se analizaron las matrices de frecuencia de ocurrencia de ítems presa por mes (M1) y para clases de talla para cada mes (M2) utilizando un análisis factorial de correspondencias (CA) (ter Braak y Smilauer 1998, Marshall y Elliott 1997). Los datos de frecuencia de ocurrencia fueron transformados logarítmicamente $(\ln (\mathrm{x}+1))$ (Jackson 1993, 1997). Los primeros tres ejes de las ordenaciones CA de los ítems presa fueron entonces comparados utilizando el análisis de Procrustes y la prueba de aleatorización (PROTEST) con la aplicación de 10,000 permutaciones al azar (Jackson 1995, Peres-Neto y Jackson 2001). El PROTEST permite analizar la concordancia en la ordenación multivariada y el valor $\mathrm{m}_{12}$ es un buen ajuste estadístico. Valores pequeños de $\mathrm{m}_{12}$ indican alta concordancia entre las matrices.

\section{RESULTADOS}

La variación en la temperatura del agua describió un ciclo estacional a lo largo del período de estudio. Las temperaturas disminuyeron de $19^{\circ} \mathrm{C}$ en abril (otoño austral) a $11^{\circ} \mathrm{C}$ en agosto (invierno austral), seguidas de un incremento de $12.5^{\circ} \mathrm{C}$ a $25.5^{\circ} \mathrm{C}$ en la primavera austral (septiembre a noviembre 1998). Por otro lado, la salinidad del agua fue 
1993, 1997). The first three axes of CA ordinations of the prey items were then compared using Procrustes analysis and randomization tests (PROTEST) with the application of 10,000 random permutations (Jackson 1995, Peres-Neto and Jackson 2001). PROTEST permits analysis of the concordance in multivariate ordination and uses $\mathrm{m}_{12}$-value as a goodness-of-fit statistic. Small values of $\mathrm{m}_{12}$ indicate high concordance between matrices.

\section{RESULTS}

Variation in water temperatures described seasonal cycles during the study period. Temperatures decreased from $19^{\circ} \mathrm{C}$ in April (austral autumn) to $11^{\circ} \mathrm{C}$ in August (austral winter), followed by an increase in the austral spring (September to November 1998), from 12.5 to $25.5^{\circ} \mathrm{C}$. Alternatively, water salinity was highly variable during the study period (fig 2), with peaks in February, April, June, August, and October related to the sand bar opening.

Stomach contents of 2915 individuals ranging from 15.6 to $42.0 \mathrm{~cm}$ TL were analyzed. Among the individuals sampled, $56.47 \%(n=1646)$ had non-empty stomachs. The availability of individuals at the sampling point differed during the study period. Significant differences $(P<0.05)$ in the proportion of stomachs with and without food were found from February to August 1998, while individuals with empty stomachs showed significant differences $(P<0.05)$ for September and November-December 1998. The degree of stomach fullness showed a regular feeding pattern, peaking from April to August (table 1). The FI values varied from 0.83 (April 1998) to 0.27 (November 1998) (table 1), peaking between February and August 1998 and declining between September and November 1998 (fig. 2).

A total of six food categories, generally in fresh condition or at the beginning of digestion, were identified (table 2). The diet was largely composed of crustaceans, polychaetes, and vegetal debris, while other food categories (molluscs, juvenile fishes, and unidentified items) represented less than $5 \%$ of the total stomach contents sampled (table 2). Crustaceans were dominant in the diet from November 1997 to March 1998 and from November to December 1998, whereas polychaetes were dominant from April to October 1998 and from January to February 1999 (fig. 3).

Fishes had a low $I_{\mathrm{G}}$ and higher FI between April and August (fig. 4). In the spring (September to November 1998), gonads matured gradually ( $I_{\mathrm{G}}$ increased), with FI values between 0.39 (September) and 0.27 (November) (fig. 4). Significant inverse relationships between $I_{\mathrm{G}}$ and FI were found for males and females $\left(r_{s}=-0.54, P=0.03\right.$, and $r_{s}=$ $-0.59, P=0.015$, respectively) and between water temperature and FI $\left(r_{s}=-0.67, P=0.004\right)$, while a non-significant relationship was found between salinity and FI $\left(r_{s}=0.073\right.$, $P=0.78)$.

We rejected the null hypothesis $(P<0.05)$ that the frequency of food consumption was independent of length
Table 2. Diet composition of Micropogonias furnieri collected in Rocha Lagoon. FO = frequency of occurrence in number and percentage (polychaetes were identified to species level but not counted by species).

Tabla 2. Composición de dieta de Micropogonias furnieri recolectada en la laguna costera de Rocha. $\mathrm{FO}=$ frecuencia de ocurrencia en número y porcentaje (los poliquetos fueron identificados solamente para conocer las especies, pero no fueron contabilizados por especie).

\begin{tabular}{lrr}
\hline Food items & FO & \%FO \\
\hline Crustacea & $\mathbf{8 0 6}$ & $\mathbf{4 8 . 9 7}$ \\
$\quad$ Cyrtograpsus angulatus & 683 & 41.49 \\
Anfipoda & 77 & 4.68 \\
$\quad$ Farfantopenaeus paulensis & 34 & 2.06 \\
Isopoda & 7 & 0.42 \\
Callinectes spp. & 5 & 0.30 \\
Polychaeta & & \\
$\quad$ Heteromastus similis & $\mathbf{4 2 1}$ & $\mathbf{2 5 . 5 7}$ \\
$\quad$ Nephtys fluviatilis & & \\
Laeonereis culveri & &
\end{tabular}

\begin{tabular}{lrr} 
Vegetal debris & $\mathbf{2 4 8}$ & $\mathbf{1 5 . 0 7}$ \\
Mollusca & $\mathbf{7 6}$ & $\mathbf{4 . 6 1}$ \\
$\quad$ Erodona mactroides & 37 & 2.25 \\
Mytella charruana & 31 & 1.88 \\
Littoridina australis & 7 & 0.42 \\
Tagelus plebeius & 1 & 0.06 \\
Pisces & $\mathbf{7 2}$ & $\mathbf{4 . 3 7}$ \\
$\quad$ Odontesthes argentinensis & 24 & 1.46 \\
Brevoortia aurea & 19 & 1.15 \\
Unidentified fishes & 17 & 1.03 \\
Jenynsea lineata & 10 & 0.61 \\
Mugil sp. & 2 & 0.12 \\
Unidentified items & $\mathbf{2 3}$ & $\mathbf{1 . 3 9}$ \\
\hline
\end{tabular}

muy variable a lo largo del período de estudio (fig. 2), con picos en febrero, abril, junio, agosto y octubre que estuvieron relacionados con la apertura de la barra de arena.

Fueron analizados los estómagos de 2915 individuos que midieron entre 15.6 y $42.0 \mathrm{~cm}$ LT. Entre los individuos muestreados, $56.47 \%(n=1646)$ presentaron contenidos en sus estómagos. La disponibilidad de los individuos en el punto de muestreo fue diferente a lo largo del período de estudio. Se encontraron diferencias significativas $(P<0.05)$ entre la proporción de estómagos con y sin alimento desde febrero a agosto de 1998, mientras que los individuos con estómagos vacíos mostraron diferencias significativas $(P<0.05)$ para septiembre y noviembre-diciembre de 1998. El grado de estómagos llenos mostró un patrón regular con altos números 


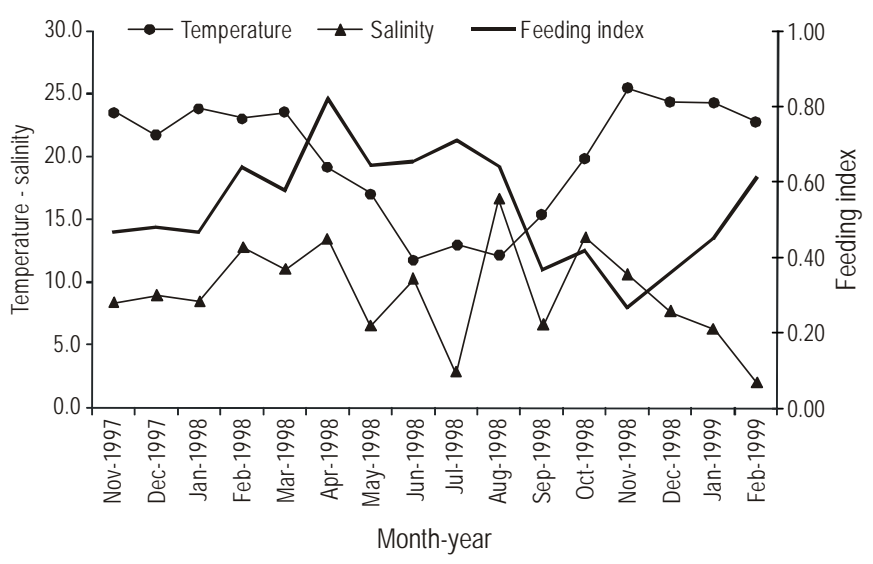

Figure 2. Monthly variation of the feeding index with respect to temperature and salinity.

Figura 2. Variación mensual del índice de alimentación con respecto a la temperatura y salinidad.

class (G-test $\left.=379.46 ; \chi^{2}{ }_{25 ; 0.05}=37.65\right)$. In general, individuals smaller than $25 \mathrm{~cm}$ fed principally on polychaetes and crustaceans, whereas individuals larger than $30 \mathrm{~cm}$ preyed on crustaceans and molluscs. Fishes and vegetal debris were found across all length classes, except in classes 5 $(36-40 \mathrm{~cm})$ and $6(41-45 \mathrm{~cm})$ (fig. 5). According to the Morisita index, there were 12 values $>0.6$, indicating high overlap between length classes (table 3 ). The lowest values were between length class $1(15-20 \mathrm{~cm})$ and classes with individuals greater than $30 \mathrm{~cm}$ (table 3 ).

The first two axes of CA for matrices M1 and M2 explained $76.4 \%$ and $53.1 \%$ of the total variance, respectively (table 4). Molluscs, fishes, and polychaetes contributed with major scores to the first factor in M1, while the same

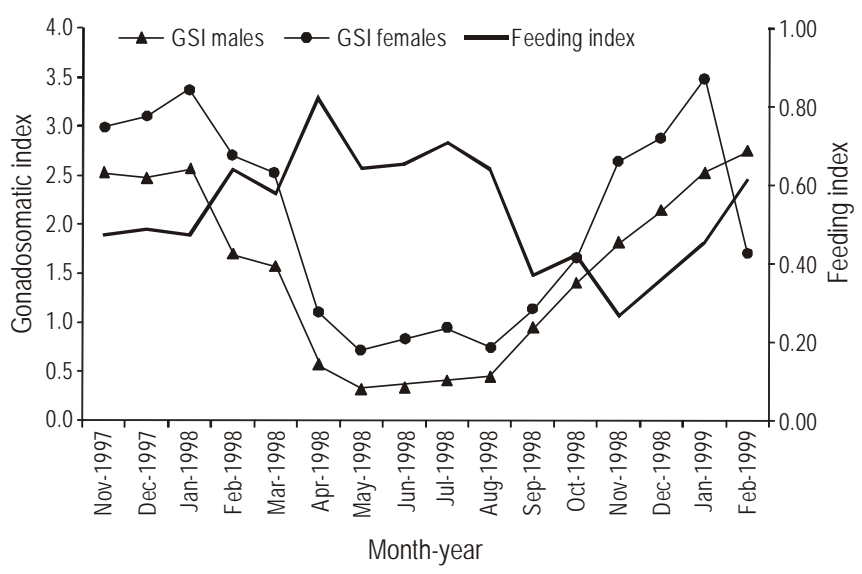

Figure 4. Monthly variation of the feeding index with respect to the gonadosomatic index by sexes (gonadosomatic index taken from Vizziano et al. 2002).

Figura 4. Variación mensual del índice de alimentación con respecto al índice gonadosomático por sexos (índice gonadosomático tomado de Vizziano et al. 2002).

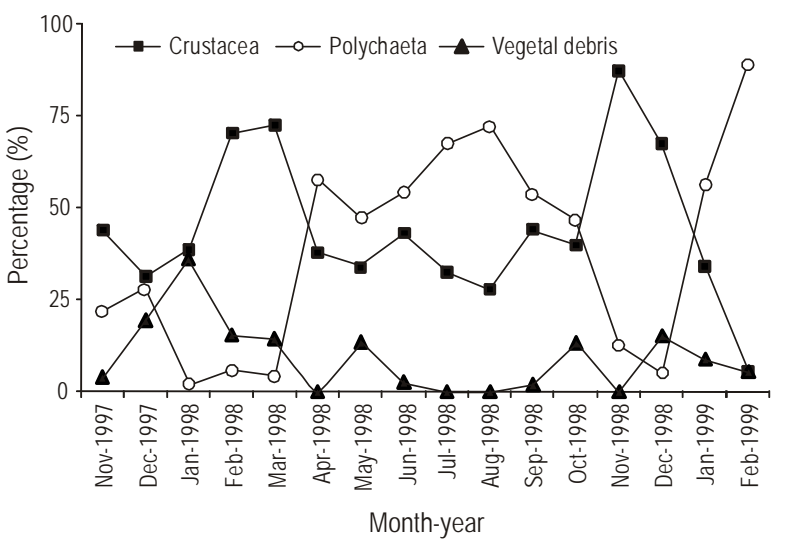

Figure 3. Monthly variation of the principal food items.

Figura 3. Variación mensual de los ítems alimenticios principales.

de estómagos llenos desde abril hasta agosto (tabla 1). Los FI variaron entre 0.83 (abril 1998) y 0.27 (noviembre 1998) (tabla 1), con valores más altos entre febrero y agosto 1998 y una tendencia decreciente entre septiembre y noviembre de 1998 (fig. 2).

Un total de seis categorías de alimento fueron identificadas, generalmente en condiciones frescas o al comienzo de la digestión (tabla 2). La dieta estuvo compuesta principalmente de crustáceos, poliquetos y restos vegetales, mientras que otras categorías de alimento (moluscos, peces juveniles, e ítems sin identificar) representaron menos que el 5\% del contenido estomacal muestreado (tabla 2). Los crustáceos fueron dominantes en la dieta desde noviembre de 1997 hasta marzo de 1998 y en noviembre y diciembre de 1998, mientras que los poliquetos fueron dominantes desde abril hasta octubre de 1998 y en enero y febrero de 1999 (fig. 3).

Los peces mostraron $I_{G}$ bajos y FI altos entre abril y agosto (fig. 4). En la primavera (septiembre a noviembre de $1998)$ las gónadas maduran gradualmente $\left(I_{G}\right.$ aumenta), con valores de FI entre 0.39 (septiembre) y 0.27 (noviembre) (fig. 4). Las relaciones inversas entre $I_{\mathrm{G}} \mathrm{y}$ FI para machos $\mathrm{y}$ hembras fueron significativas $\left(r_{s}=-0.54, P=0.03 \mathrm{y}\right.$ $r_{s}=-0.59, P=0.015$, respectivamente) y entre la temperatura del agua y FI $\left(r_{s}=-0.67, P=0.004\right)$, mientras que las relaciones entre salinidad y FI no fueron significativas $\left(r_{s}=0.073\right.$, $P=0.78>0.05$ ).

Se rechazó la hipótesis nula $(P<0.05)$ de que la frecuencia de la ingesta de alimento es independiente de las clases de talla (prueba de $\mathrm{G}=379.46 ; \chi^{2} 25 ; 0.05=37.65$ ). En general, los individuos menores que $25 \mathrm{~cm}$ se alimentaron principalmente de poliquetos y crustáceos, mientras que los individuos mayores que $30 \mathrm{~cm}$ depredaron sobre crustáceos y moluscos. Los peces y los restos vegetales fueron encontrados en todas las clases de talla, excepto en las clases $5(36-40 \mathrm{~cm}) \mathrm{y}$ 6 (41-45 cm) (fig. 5). El índice de Morisita mostró 12 valores mayores que 0.6 , indicando altas superposiciones entre clases de talla (tabla 3). Los valores menores fueron entre las clases 


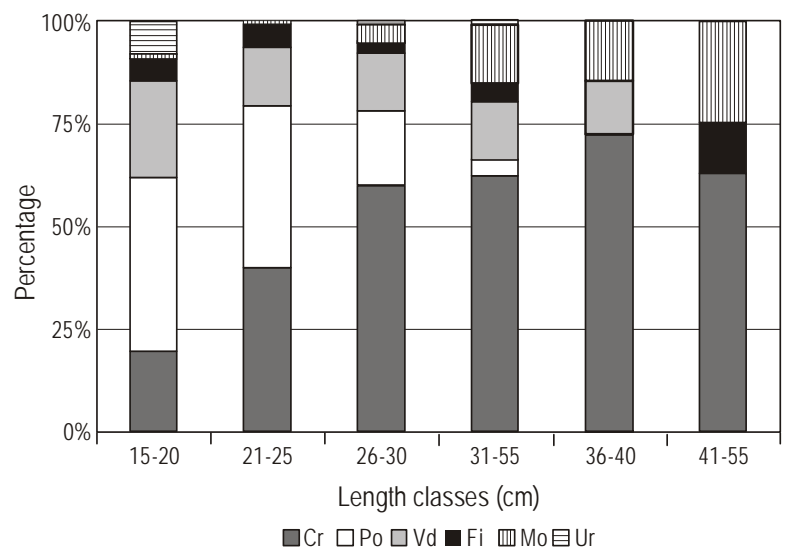

Figure 5. Variation of food items by length groups $(\mathrm{Cr}=$ crustaceans, $\mathrm{Po}=$ polychaetes, $\mathrm{Vd}=$ vegetal debris, $\mathrm{Fi}=$ fishes, $\mathrm{Mo}=$ molluscs, $\mathrm{Ur}=$ unidentified remains).

Figura 5. Variación de los ítems alimenticios principales por grupo de tallas $(\mathrm{Cr}=$ crustáceos, $\mathrm{Po}=$ poliquetos, $\mathrm{Vd}=$ restos vegetales, $\mathrm{Fi}=$ peces, $\mathrm{Mo}=$ moluscos, $\mathrm{Ur}=$ restos no identificados).

variables plus proportion of empty stomachs contributed to M2. Unidentified items contributed with major scores to the second factor for both matrices. Crustaceans and vegetal debris had minor contributions (table 4). In M1, negative scores for factor 1 were associated with November-December 1997 and January-March 1998, while positive scores were associated with the remaining months. The CA of M2 showed a gradual change in length classes between negative and positive scores for factor 1 (results not shown). The ordinations of the two matrices were significantly concordant $\left(\mathrm{m}_{12}=0.1200, P=0.0029\right)$.

\section{DisCUSSION}

This study was conducted in the area of the lagoon most influenced by marine coastal water and where the abundance of $M$. furnieri is highest. Although migration patterns within the lagoon are poorly understood, it is known that fishes get closer to the coast at sunset and that the gear used catches most of the size classes for individuals living in the lagoon (Saona et al. 2003). de talla $1(15-20 \mathrm{~cm})$ y las de individuos mayores que $30 \mathrm{~cm}$ (tabla 3).

Los primeros dos ejes de CA para las matrices M1 y M2 explicaron un $76.36 \%$ y un $53.13 \%$ de la varianza total, respectivamente (tabla 4). Los moluscos, los peces y los poliquetos contribuyeron con mayores factores de carga al primer factor en la M1, y las mismas variables más los estómagos vacíos en la M2. Los restos no identificados contribuyeron con los mayores valores al segundo factor en ambas matrices. Los crustáceos y los restos vegetales presentaron contribuciones menores (tabla 4). En M1, los valores negativos para el factor 1 estuvieron asociados con los meses de noviembre a diciembre de 1997 y de enero a marzo de 1998, y los valores positivos estuvieron asociados con el resto de los meses. El CA de la M2 mostró un cambio gradual de clases de talla entre valores positivos y negativos para el factor 1 (resultados no mostrados). Las ordenaciones de las dos matrices fueron significativamente concordantes $\left(\mathrm{m}_{12}=0.1200, P=0.0029\right)$.

\section{DiscUSIÓN}

Este estudio fue realizado en el área de la laguna con mayor influencia de agua marina costera y donde la disponibilidad y abundancia de la corvina blanca es mayor. Si bien el patrón de migración dentro de la laguna es poco conocido, al atardecer los peces se acercan a la costa y las redes utilizadas capturan la mayoría de los intervalos de talla de los individuos que viven en la laguna (Saona et al. 2003).

Nuestro estudio confirma la importancia de los crustáceos decápodos (principalmente Cyrtograpsus angulatus), poliquetos y restos vegetales en la dieta de $M$. furnieri en la laguna de Rocha. La importancia de los crustáceos y poliquetos en la dieta de M. furnieri es bien conocida (Puig 1986, Vazzoler 1991, Sánchez et al. 1991, Masello et al. 2002). La baja frecuencia de anfípodos e isópodos observada en la dieta se debe probablemente a la ausencia de especímenes de $M$. furnieri menores que $14 \mathrm{~cm}$ en las muestras (Mandali y Paes 1998, 2005; Hozbor y García de la Rosa 2000). También los peces juveniles fueron ocasionales en la dieta, y resultados similares fueron encontrados para la corvina blanca que habita en el estuario del Río de la Plata (Puig 1986, Sánchez et al. 1991, Masello et al. 2002). A pesar de la diferencia

Table 3. Diet overlap by length classes (cm) for Micropogonias furnieri in Rocha Lagoon.

Tabla 3. Superposición de dieta por clase de talla (cm) para Micropogonias furnieri en la laguna costera de Rocha.

\begin{tabular}{|c|c|c|c|c|c|c|}
\hline Length class & $15-20$ & $21-25$ & $26-30$ & $31-35$ & $36-40$ & $41-45$ \\
\hline $15-20$ & 1.00 & & & & & \\
\hline 21-25 & 0.91 & 1.00 & & & & \\
\hline 26-30 & 0.66 & 0.89 & 1.00 & & & \\
\hline 31-35 & 0.49 & 0.75 & 0.96 & 1.00 & & \\
\hline $36-40$ & 0.37 & 0.66 & 0.91 & 0.98 & 1.00 & \\
\hline $41-45$ & 0.35 & 0.64 & 0.88 & 0.96 & 0.97 & 1.00 \\
\hline
\end{tabular}


Table 4. Results of the correspondence analysis for prey items with respect to Matrix 1 and Matrix 2 (see materials and methods). Contributions of months (M1) and length classes by month (M2) are not shown.

Tabla 4. Resultados del análisis de correspondencia para ítems presa con respecto a la Matriz 1 y Matriz 2 (ver materiales y métodos). La contribución de meses (M1) y las clases de talla por mes (M2) no se muestran.

\begin{tabular}{|c|c|c|c|c|}
\hline & \multicolumn{2}{|c|}{ Matrix 1} & \multicolumn{2}{|c|}{ Matrix 2} \\
\hline Singular values & 0.39 & 0.27 & 0.44 & 0.33 \\
\hline Eigenvalues & 0.15 & 0.07 & 0.20 & 0.11 \\
\hline$\%$ inertia & 51.93 & 24.44 & 34.30 & 18.83 \\
\hline Cumulative \% inertia & 51.93 & 76.37 & 34.30 & 53.13 \\
\hline Fishes & -0.96 & 0.12 & 0.83 & -0.53 \\
\hline Molluscs & -0.85 & 0.56 & 1.18 & -0.51 \\
\hline Vegetal debris & -0.33 & -0.19 & 0.37 & -0.04 \\
\hline Unidentified items & -0.29 & -0.95 & 0.29 & 0.85 \\
\hline
\end{tabular}

Our study confirms the importance of decapod crustaceans (principally Cyrtograpsus angulatus), polychaetes, and vegetal debris in the diet of $M$. furnieri in Rocha Lagoon. The importance of crustaceans and polychaetes in the diet of this species is well known (Puig 1986, Vazzoler 1991, Sánchez et al. 1991, Masello et al. 2002). The low frequency of amphipods and isopods in the diet observed in this study is probably due to the absence of specimens of $M$. furnieri smaller than $14 \mathrm{~cm}$ in our samples (Mandali and Paes 1998, 2005; Hozbor and García de la Rosa 2000). Juvenile fishes were also occasional in the diet, in concordance with the diet of whitemouth croaker inhabiting the Río de la Plata estuary (Puig 1986, Sánchez et al. 1991, Masello et al. 2002). Despite the variation in form and size of the principal prey items, there was strong selection for certain prey within the year, in relation to other available benthic prey items (i.e., molluscs). Despite the fact that molluscs (Erodona mactroides and Heleobia australis) are abundant in the benthic community throughout the year in Rocha Lagoon (Jorcin 1996), they were only occasionally ingested by $M$. furnieri here, a result also found in Patos Lagoon, Brazil (Mandali and Paes 1998). In this study, M. furnieri appears to select the largest available organisms. Such behavior is consistent with the optimal foraging theory that predicts fish feeding on optimal prey for higher net gains (Stephens and Krebs 1986).

Temporal changes in the diet of $M$. furnieri could indicate changes in the availability of organisms despite the abundance of $C$. angulatus and polychaetes in the system (Jorcin 1996). Many authors define the whitemouth croaker as an opportunistic benthic predator (Sánchez et al. 1991, Mandali relativa entre la forma y la talla de las presas principales, hubo una fuerte selección a lo largo del año, en relación con otras presas bentónicas que estaban disponibles (i.e., moluscos). Los moluscos (Erodona mactroides y Heleobia australis) son abundantes en la comunidad bentónica de la laguna de Rocha durante todo el año (Jorcin 1996); sin embargo, esos ítems presa fueron ingeridos solo ocasionalmente por $M$. furnieri, como fue encontrado en la laguna de los Patos, Brasil (Mandali y Paes 1998). En este estudio, parecería que $M$. furnieri elige los organismos más grandes disponibles. Esto es consistente con la teoría de forrajeo óptimo que predice una mayor ganancia neta para peces que se alimentan de presas de tamaño óptimo (Stephens y Krebs 1986).

Los cambios temporales en la dieta de $M$. furnieri pueden ser vistos como cambios en la disponibilidad de organismos, a pesar de que los cangrejos (C. angulatus) y los poliquetos son especies permanentes y abundantes en el sistema (Jorcin 1996). Muchos autores definen a esta especie como un depredador bentónico oportunista (Sánchez et al. 1991, Mandali y Paes 1998, Hozbor y García de la Rosa 2000) que se alimenta de la presa más abundante presente en el sistema. Resultados similares fueron encontrados para Micropogonias undulatus (Chao y Musick 1977, Overstreet y Heard 1978). La corvina blanca se alimenta intensamente entre febrero (final del verano austral) y agosto (invierno austral), cuando la temperatura del agua disminuye y los peces entran en una fase de reposo, con bajos índices gonadosomáticos y con las gónadas inmaduras, en desarrollo o en reposo (Vizziano et al. 2002). Los poliquetos fueron el ítem presa predominante antes del período reproductivo (abril a octubre), y esos organismos 
and Paes 1998, Hozbor and García de la Rosa 2000), feeding on the most abundant prey items present in the system. Similar results were found for Micropogonias undulatus (Chao and Musick 1977, Overstreet and Heard 1978). The whitemouth croaker feeds intensively between February (late austral summer) and August (austral winter), when water temperature declines and fishes enter a resting phase, displaying a low gonadosomatic index and immature, developing or resting gonads (Vizziano et al. 2002). The predominant food item ingested before the reproductive period (April-October) was polychaetes, which include organisms that contain high levels of protein, lipids, and highly unsaturated fatty acids (HUFA) (Orlando and Passos 1995, GarcíaAlonso et al. 2008). Many authors have found that the ovarian lipids of fishes also contain high proportions of HUFA (Singh and Singh 1979, 1984; Mourente et al. 2001; Sorbera et al. 2001), implying that the availability of polychaetes could play an important role for whitemouth croaker reproduction. Size at first maturity for both sexes in Rocha Lagoon is $20 \mathrm{~cm}$ (Vizziano et al. 2002) and polychaetes were predominant in fishes smaller than $25 \mathrm{~cm}$. Such feeding habits agree more with a selective predator than with an opportunistic generalist predator (Delbeek and Williams 1988).

This species spawns during late spring and summer when fish have a high gonadosomatic index and individuals are in gravid and running gonad stages (Vizziano et al. 2002). Thus, the large investment of energy in gonad development suggests that the whitemouth croaker adopts a selective foraging strategy that maximizes prey quality. Such a strategy could provide the whitemouth croaker with adequate resources to meet energetic demands when prey are numerous, particularly during intensive feeding periods in the spawning season (when 50\% of the individuals presented stomachs with food), as also described for Río de la Plata coastal waters in summer (Puig 1986, Masello et al. 2002). In the Patos Lagoon estuary, the high stomach fullness and the presence of nondigested food items suggest a strategy of continuous food ingestion (Mandali and Paes 1998, 2005).

The importance of different food items varies with fish size and season (Puig 1986, Sánchez et al. 1991, Masello et al. 2002, this work), with large overlap between length classes. This may result in increased intraspecific competition leading to the establishment of spatial segregation between length classes.

In addition to prey availability and the physical characteristics of Rocha Lagoon, predatory pressure and intraspecific competition may play an important role in determining the distribution of fish species. Nonetheless, the simultaneous occurrence of different size groups during spawning activities (Vizziano et al. 2002) and the spatial segregation between the length classes within the lagoon (Saona et al. 2003) suggest low intraspecific competition, but more studies are required to verify this hypothesis. contienen altos niveles de proteínas, lípidos y ácidos grasos altamente insaturados (HUFA) (Orlando y Passos 1995, Garcia-Alonso et al. 2008). Muchos autores encontraron que los lípidos en los ovarios de los peces contienen altas proporciones de HUFA (Singh y Singh 1979, 1984; Mourente et al. 2001; Sorbera et al. 2001), implicando que la disponibilidad de poliquetos podría jugar un rol importante en la reproducción de la corvina blanca. La talla de primera madurez para ambos sexos en la laguna de Rocha es de $20 \mathrm{~cm}$ (Vizziano et al. 2002), y los poliquetos fueron predominantes en los peces menores que $25 \mathrm{~cm}$. Tales hábitos de alimentación coinciden más con un depredador de comportamiento selectivo que con un depredador generalista-oportunista (Delbeek y Williams 1988).

El desove de esta especie ocurre hacia fines de la primavera y verano austral, cuando los peces presentan altos índices gonadosomáticos y estados de desarrollo gonadales maduros y grávidos (Vizziano et al. 2002). Así la gran inversión de energía en el desarrollo gonadal sugiere que la corvina blanca adopta una estrategia de alimentación selectiva que maximiza la calidad de la presa. Tal estrategia podría proveer a la corvina con recursos adecuados para satisfacer las demandas energéticas cuando las presas son numerosas, particularmente durante periodos de alimentación intensiva en la estación de desove (cuando 50\% de los individuos presentaron estómagos con alimento), como ha sido descrito para las aguas costeras del Río de la Plata en verano (Puig 1986, Masello et al. 2002). En el estuario de la laguna de los Patos, un número alto de estómagos llenos y la presencia de ítems de alimentación no digeridos totalmente sugieren una estrategia de ingestión de alimento continua (Mandali y Paes 1998, 2005).

La importancia de los diferentes ítems alimenticios varía con la talla del pez y con la época del año (Puig 1986, Sánchez et al. 1991, Masello et al. 2002, este trabajo), con grandes superposiciones entre clases de longitud. Esto puede resultar en un aumento de la competencia intraespecífica que conduzca al establecimiento de una segregación espacial entre grupos de talla.

La presión de depredación y la competencia intraespecífica, junto con la variación de la disponibilidad de presas y las características físicas de la laguna de Rocha, pueden jugar un rol importante en determinar la distribución de los peces. Sin embargo, la ocurrencia simultanea de diferentes grupos de talla durante las actividades de desove (Vizziano et al. 2002) y la segregación espacial de los grupos de talla dentro de la laguna (Saona et al. 2003) sugieren una baja competencia intraespecífica, pero se requieren más estudios para verificar esta hipótesis.

En general, los peces que habitan los estuarios no tienen dietas altamente especializadas (Edgar y Shaw 1995, Chaves y Umbria 2003). De todas formas, los hábitos temporales de alimentación de $M$. furnieri en la laguna de Rocha mostraron un uso selectivo y apropiado de las presas, de acuerdo con 
In general, fishes that inhabit estuaries do not have highly specialized diets (Edgar and Shaw 1995, Chaves and Umbria 2003). Nonetheless, the temporal feeding habits of $M$. furnieri in Rocha Lagoon showed a selective and appropriate utilization of prey items, according to the reproductive cycle of the species and annual variability in water temperature. Ecosystems such as this coastal lagoon are highly productive (Bonilla et al. 2006) and constitute areas relevant for conservation (Díaz et al. 1999). An analysis of the dominant processes (i.e., sediment resuspension and hydrological exchange with the ocean) has determined the temporal and spatial dynamics of biota across diverse temporal scales and revealed the importance of the benthic compartment (microphytobenthos and zoobenthos) in the lagoon (Bonilla et al. 2006). Recent land-use changes occurring in the basin, as well as ongoing eutrophication and the artificial modification of the frequency of connection with the ocean, could work synergistically to affect the ecological functioning of this lagoon (Bonilla et al. 2006) and in consequence the prey availability for benthic fishes such as $M$. furnieri.

\section{ACKNOWLEDGMENTS}

Funding was provided by PROBIDES (URU/97/G31 project PNUD-GEF-MVTOMA) and the Faculty of Sciences (Universidad de la República, Uruguay). We are very grateful to Denise Vizziano for assistance with sampling efforts and to the local fishermen of Rocha Lagoon for their invaluable help with field sampling: Vicente, José, Jorge, and Santiago Ballesteros; Fabián Huelmo; Angel Alvarez; and Walter Fernández. We also thank Fabricio Scarabino (DINARA and Museo Nacional de Historia Natural, Montevideo) for improving the benthos nomenclature.

\section{REFERENCES}

Araujo FG. 1984. Feeding habits of three marine catfishes (Ariidae) in Lagoa dos Patos estuary (RS), Brasil. Atlantica 7: 47-63.

Arena G. 1990. Maximum sustainable yield of the white croaker (Micropogonias furnieri) in the operating area of the Uruguayan fleet, through surplus production models. Frente Marit. 7: 25-36.

Bonilla S, Conde D, Aubriot L, Rodríguez-Gallego L, Piccini C, Meeroff E, Rodríguez-Graña L, Calliari D, Gómez P, Machado I, Britos A. 2006. Structured process of biological communities in coastal lagoons of Uruguay. In: Menafra R, RodríguezGallego L, Scarabino F, Conde D (eds.), Bases para la Conservación y el Manejo de la Costa Uruguaya. Vida Silvestre Uruguay, Montevideo, pp. 611-630.

Bozec YM, Gascuel D, Kulbicki M. 2004. Trophic model of lagoonal communities in a large open atoll (Uvea, Loyalty islands, New Caledonia). Aquat. Living Resour. 17: 151-162. http://dx.doi.org/10.1051/alr:2004024

Castello JP. 1985. La ecología de los consumidores del estuario de la Laguna dos Patos, Brasil. In: Yáñez-Arancibia A (ed.), Fish Community Ecology in Estuaries and Coastal Lagoons: Towards an Ecosystem Integration. Universidad Nacional Autónoma de México, México, pp. 383-406. el ciclo reproductivo de la especie y la variabilidad de la temperatura del agua a lo largo del año. El ecosistema de la laguna costera de Rocha es altamente productivo (Bonilla et al. 2006) y posee áreas relevantes para la conservación (Díaz et al. 1999). El análisis de los procesos dominantes (resuspensión de sedimentos e intercambio hidrológico con el océano) determina la dinámica temporal y espacial de la biota a lo largo de diversas escalas temporales y revela la importancia del compartimiento bentónico (microfitobentos y zoobentos) en la laguna (Bonilla et al. 2006). Los cambios recientes en el uso del suelo en la cuenca, la eutrofización y la modificación artificial de la frecuencia de conexión con el océano podrían afectar la sinergia del funcionamiento ecológico de esta laguna (Bonilla et al. 2006) y, como consecuencia, la disponibilidad de presas para los peces bentónicos como M. furnieri.

\section{Agradecimientos}

Los autores agradecen los fondos proporcionados por PROBIDES (URU/97/G31 proyecto PNUD-GEFMVTOMA) y la Facultad de Ciencias (Universidad de la República, Uruguay). Agradecemos a Denise Vizziano su asistencia en la tarea de muestreo y a los pescadores artesanales de la laguna de Rocha (Vicente, José, Jorge y Santiago Ballesteros; Fabián Huelmo, Angel Alvarez; y Walter Fernández) su valiosa ayuda y colaboración en los muestreos. También agradecemos a Fabricio Scarabino (DINARA y Museo Nacional de Historia Natural, Uruguay) por la revisión de la nomenclatura del bentos.

Conde D, Aubriot L, Sommaruga R. 2000. Changes in UV penetration associated with marine intrusions and freshwater discharge in a shallow coastal lagoon of the Southern Atlantic Ocean. Mar. Ecol. Prog. Ser. 207: 19-31. http://dx.doi.org/10.3354/meps207019

Chao L, Musick JA. 1977. Life history, feeding habits, and functional morphology of juvenile sciaenid fishes in the York River estuary, Virginia. Fish. Bull. 75: 567-702.

Chaves PTC, Umbria SC. 2003. Changes in the diet composition of transitory fishes in coastal systems, estuary and continental shelf. Braz. Arch. Biol. Technol. 46: 41-46. http://dx.doi.org/10.1590/S1516-89132003000100007

Delbeek JC, Williams DD. 1988. Feeding selectivity of four species of sympatric stickleback in brackish water habitats in eastern Canada. J. Fish Biol. 32: 41-62. http://dx.doi.org/10.1111/j.1095-8649.1988.tb05334.x

Díaz A, Porzecansky I, Rilla F, Casciani M, Hernández J, Acosta J, Iglesias A, Sciandro J, Azpiroz A, Baez F, Molina B, Prigioni C, Rodríguez R, Bazzani S, Fagetti C, Pérez J, Torres A, Guarino P, Castillo A, Fernández S, Jaurena M, Norbis W, Vitancurt J. 1999. Management Plan. Biosphere Reserve Bañados del Este, Uruguay. Editorial Mosca Hnos., Montevideo, Uruguay, 159 pp. 
Edgar G, Shaw C. 1995. The production and trophic ecology of shallow water fish assemblages in southern Australia. II. Diets of fishes and trophic relationships between fishes and benthos at Western Port, Victoria. J. Exp. Mar. Biol. Ecol. 194: 83-106. http://dx.doi.org/10.1016/0022-0981(95)00084-4

García-Alonso J, Müller CT, Hardege JD. 2008. Influence of food regimes and seasonality on fatty acid composition in the ragworm. Aquat. Biol. 4: 7-13. http://dx.doi.org/10.3354/ab00090

Goncalves AA, Feijò JA, Paes J. 1999. Trophic description of first life stages of Micropogonias furnieri (Sciaenidae) in Lagoa dos Patos estuary, RS, Brazil. Atlantica 21: 93-103.

Hozbor NM, García de la Rosa MB. 2000. Food of juvenile croaker (Micropogonias furnieri) in the Mar Chiquita coastal lagoon (Buenos Aires, Argentina). Frente Marit. 18: 59-70.

Hureau J. 1970. Biologie comparée de quelques poissons antarctiques (Nototheniidae). Bull. Inst. Oceanogr. Monaco 68: $1-244$.

Hyslop EJ. 1980. Stomach content analysis: A review of methods and their application. J. Fish Biol. 17: 411-429. http://dx.doi.org/10.1111/j.1095-8649.1980.tb02775.x

Isaac VJ. 1988. Synopsis of biological data on the whitemouth croaker Micropogonias furnieri (Desmarest, 1823). FAO Fish. Synop. No. 150: 35 pp.

Jackson DA. 1993. Multivariate analysis of benthic invertebrate communities: The implication of choosing particular data standardizations, measures of association, and ordination methods. Hydrobiologia 268: 9-26. http://dx.doi.org/10.1007/BF00005737

Jackson DA. 1995. PROTEST: A Procrustean Randomization Test of community environment concordance. Ecoscience 2: 297-303.

Jackson DA. 1997. Compositional data in community ecology: The paradigm or peril of proportions? Ecology 78: 929-940. http://dx.doi.org/10.1890/00129658(1997)078[0929:CDICET]2.0.CO;2

Jorcin A. 1996. Distribution, abundance and biomass of Erodona mactroides (Mollusca, Bivalvia, Daudin, 1801) in Rocha Lagoon (Rocha, Uruguay). Revista Brasil. Biol. 56: 155-162.

Langton RW. 1982. Diet overlap between the Atlantic cod Gadus morhua, silver hake Merluccius bilinearis and fifteen other northwest Atlantic finfish. Fish. Bull. 80: 745-759.

Mandali G, Paes J. 1998. Feeding chronology and diet of corvina, Micropogonias furnieri, in Lagoa dos Patos estuary, Rs, Brazil. Atlantica 20: 55-72.

Mandali G, Paes J. 2005. Diel feeding, daily food consumption and the predatory impact of whitemouth croaker (Micropogonias furnieri) in an estuarine environment. Mar. Ecol. 26: 130-139.

Marshall S, Elliott M. 1997. A comparison of univariate and multivariate numerical and graphical techniques for determining inter- and intraspecific feeding relationships in estuarine fish. J. Fish Biol. 51: 526-545. http://dx.doi.org/10.1111/j.1095-8649.1997.tb01510.x

Masello A, Scarabino F, Gamarra M, Menafra R. 2002. Study of feeding habits and stomach contents of Micropogonias furnieri. In: Vizziano D, Puig P, Mesones C, Nagy G (eds.), Río de la Plata research to manage the environment, fish resources and the fishery in the saline front. Ecoplata Program, Montevideo, Uruguay, pp. 147-161.

Mendoza-Carranza M, Vieira J. 2008. Whitemouth croaker Micropogonias furnieri (Desmarest 1823) feeding strategies across four southern Brazilian estuaries. Aquat. Ecol. 42: 83-93. http://dx.doi.org/10.1007/s10452-007-9084-4

Mourente G, Megina C, Díaz-Salvago E. 2001. Lipids in female northern bluefin tuna (Thunnus thynnus thynnus L.) during sexual maturation. Fish Physiol. Biochem. 24: 351-363. http://dx.doi.org/10.1023/A:1015011609017

Norbis W. 1995. Influence of wind, behavior and characteristics of the croacker (Micropogonias furnieri) artisanal fishery in the Río de la Plata (Uruguay). Fish. Res. 22: 43-58. http://dx.doi.org/10.1016/0165-7836(94)00310-S

Norbis W, Galli O. 2004. Feeding habits of the flounder Paralichthys orbignyanus (Valenciennes 1839) in a shallow coastal lagoon of the southern Atlantic Ocean: Rocha, Uruguay. Cienc. Mar. 30: 619-625.

Orlando J, Passos A. 1995. Seasonal changes in lipid content and composition of the polychaete Nereis (Hediste) diversicolor. Comp. Biochem. Physiol. B: Biochem. Mol. Biol. 111: 579-586.

Overstreet R, Heard R. 1978. Food of the Atlantic croaker, Micropogonias undulatus, from Mississippi Sound and the Gulf Mexico. Gulf Res. Rep. 6: 145-152.

Palomares MLD, Pauly D. 1999. Predicting the food consumption of fish populations as functions of mortality, food type, morphometrics, temperature and salinity. Mar. Freshwat. Res. 49: 447-453.

Peres-Neto PR, Jackson DA. 2001. How well do multivariate data sets match? The advantages of a Procrustean superimposition approach over the Mantel test. Oecologia 129: 169-178. http://dx.doi.org/10.1007/s004420100720

Pintos W, Conde D, De León R, Cardezo MJ, Jorcín A, Sommaruga R. 1991. Some limnological characteristics of Laguna de Rocha (Uruguay). Rev. Bras. Biol. 51: 79-84.

Puig P. 1986. Stomach content analysis of white croaker (Micropogon opercularis) (Sciaenidae, Perciformes), summer 1984. Publ. Com. Téc. Mix. Frente Marit. 1: 333-340.

Rodríguez-Graña L, Calliari D, Conde D, Sellanes J, Urrutia R 2008. Food web of a SW Atlantic shallow coastal lagoon: Spatial environmental variability does not impose substantial changes in the trophic structure. Mar. Ecol. Prog. Ser. 362: 69-83. http://dx.doi.org/10.3354/meps07401

Sánchez F, Mari N, Lasta C, Giangiobbe A. 1991. Feeding of the croaker (Micropogonias furnieri) in Samborombon Bay. Frente Marit. 8: 43-50.

Saona G, Forni F, Vizziano D, Norbis W. 2003. Structure by size, sex, and maturity stage of the white croaker (Micropogonias furnieri, Desmarest 1823; Teleostei: Sciaenidae) in the bycatch of the artisanal fishery at Rocha Lagoon, Uruguay. Cienc. Mar. 29: 315-324.

Singh A, Singh T. 1979. Seasonal fluctuation in lipid and cholesterol content of ovary, liver and blood serum in relation to annual sexual cycle in Heteropneustes fossilis (Bloch). Endocrinology 73: 47-54.

Singh I, Singh T. 1984. Changes in gonadotrophin, lipid and cholesterol levels during annual reproductive cycle in the freshwater teleost, Cirrhinus mrigala (Ham.). Ann. Endocrinol. 45: 131-136.

Smith P, Zaret MT. 1982. Bias in estimating niche overlap. Ecology 63: 1248-1253. http://dx.doi.org/10.2307/1938851

Sorbera L, Asturiano JF, Carrillo M, Zanuy S. 2001. Effects of polyunsaturated fatty acids and prostaglandins on oocyte maturation in a marine teleost, the European sea bass (Dicentrarchus labrax). Biol. Reprod. 64: 382-389.

Sokal RR, Rohlf FJ. 1995. Biometry: The Principles and Practice of Statistics in Biological Research. 3rd ed. WH Freeman and Company, New York, 887 pp.

Stephens DW, Krebs LR. 1986. Foraging Theory. Princeton University Press, Princeton, 153 pp.

Teixeira RL, Falcao GAF, Melo SC. 1992. Occurrency and feeding of Sciaenidae juveniles (Pisces: Perciformes) from surf zone beaches of Maceio, Brazil. Atlantica 4: 29-42. 
ter Braak CJF, Smilauer P. 1998. CANOCO Reference Manual and User's Guide to Canoco for Windows. Ver. 4. Ithaca, New York, $352 \mathrm{pp}$.

Vazzoler AE. 1991. Síntese de conhecimentos sobre a biología da corvina Micropogonias furnieri (Desmarest, 1823), da costa do Brasil. Atlántica 13: 55-74.

Vizziano D. 2002. Reproductive cycle of the white croaker Micropogonias furnieri in the frontal zone of the Río de la Plata.
In: Vizziano D, Puig P, Mesones C, Nagy G (eds.), Río de la Plata research to manage the environment, fish resources and the fishery in the saline front. Ecoplata Program, Montevideo, Uruguay, pp. 103-111.

Vizziano D, Forni F, Saona G, Norbis W. 2002. Reproduction of the white croaker Micropogonias furnieri (Pisces: Sciaenidae) in a shallow temperate coastal lagoon of the Southern Atlantic Ocean (Uruguay). J. Fish Biol. 61: 196-206.

http://dx.doi.org/10.1111/j.1095-8649.2002.tb01771.x

Received August 2012, received in revised form February 2013, accepted February 2013. 